\title{
Lettre éditoriale
}

Les années 2019 et 2020 ont vu la disparition de deux très grandes figures de la géotechnique française, Pierre Habib et Pierre Duffaut. Six sociétés savantes, l'Association française du génie parasismique, l'Association française des tunnels et de l'espace souterrain, le Comité français des barrages et réservoirs, le Comité français de géologie de l'ingénieur et de l'environnement, le Comité français de mécanique des roches, le Comité français de mécanique des sols et de géotechnique, dont Pierre Habib et Pierre Duffaut avaient été des membres ou présidents éminents, se sont associées pour organiser une journée d'hommage commune. La pandémie qui a frappé le monde a différé à plusieurs reprises, jusqu'au 15 octobre 2021, la tenue de cette journée.

Les présidents des six comités ont apporté un soutien chaleureux à cette initiative; leur concours a permis de lever efficacement divers obstacles. Une petite équipe issue du conseil du CFMR a pris en charge l'organisation de la journée ; elle doit être remerciée, comme tous ceux qui ont apporté sous une forme ou une autre une aide précieuse. Farimah Masrouri, rédactrice en chef de la Revue française de géotechnique, a accepté avec enthousiasme que soit consacré à la mémoire de Pierre Habib et Pierre Duffaut un numéro spécial de la Revue. Pierre Habib l'avait créée en 1977 et dirigée pendant de longues années; tous deux ont contribué à son succès par de nombreux articles.

Ce numéro présente donc un caractère particulier. Après une évocation de leur œuvre et de leur personnalité par Jean Salençon et Jean-Louis Giafferi, huit géotechniciens, choisis par les présidents des comités, abordent une question scientifique en évoquant les apports de Pierre Habib et Pierre Duffaut et les développements qu'ils ont engendrés.

Jean Salençon évoque le Laboratoire de mécanique des solides (LMS), créé par Jean Mandel, dont Pierre Habib, « ingénieur et homme de science », remarquable pédagogue, a été la poutre maîtresse pendant près de 50 ans. Il rappelle la profonde influence qu'a eue sur lui Armand Meyer, autre immense mécanicien des roches. Il illustre ses apports dans la compréhension de l'effet d'échelle et de la dualité glissement et grain dans les phénomènes de rupture, un thème sans cesse remis sur le métier de son inspiration. Il évoque son humanisme, discret et universel, dont sont témoins ceux qui l'ont connu de près, l'enveloppe corporelle ne signale rien de l'intelligence de chaque homme.

Jean-Louis Giafferi met en exergue l'apport à l'art des tunnels et des barrages, au milieu $\mathrm{du}>\mathrm{xx}^{\mathrm{e}}$ siècle, de la géologie, et de l'hydrogéologie, dont la curiosité multidisciplinaire de Pierre Duffaut aura été un intermédiaire éclairé. Il retrace sa carrière au service géologique d'EDF, puis au BRGM, consacrée aux tunnels et aux barrages. Il rappelle le traumatisme qu'a constitué pour la génération d'ingénieurs à laquelle appartiennent les deux Pierre la terrible catastrophe de Malpasset en 1959. La notion de retraite s'applique mal à
Pierre Duffaut - et d'ailleurs à Pierre Habib. Jean-Louis Giafferi rappelle que ce qu'il vaut mieux appeler la seconde partie de son activité professionnelle a permis à Pierre Duffaut de présider à l'élaboration d'une œuvre collective, la collection des Manuels de Mécanique des Roches, qui a couronné son œuvre de pédagogue et de transmetteur.

Marc Panet et Jean Sulem, éminents contributeurs à la méthode convergence-confinement (ou d'offre et de demande de soutènement, pour reprendre la terminologie de Pierre Duffaut, grand créateur de concepts), évoquent l'important apport des chercheurs du LMS, sous l'impulsion personnelle de Pierre Habib, à la question centrale du taux de déconfinement.

Pierre Bérest évoque les souvenirs d'un jeune chercheur que Pierre Habib remplaça au pied levé pour conduire sur des cavités de stockage de Gaz de France un essai original de mesures d'oscillations, trop longues pour avoir été jusque-là identifiées. Il dit leur émotion partagée devant la coïncidence des prévisions du calcul en aveugle avec des observations qui étaient les premières du genre.

Dominique Allagnat souligne l'importance de l'application rigoureuse des recommandations pour la mise en œuvre des tirants d'ancrage, dont la formulation a débuté avec Pierre Habib dans les années 1970, et en donne plusieurs illustrations récentes.

Jean Sulem rappelle que l'exemple du Vajont avait suggéré à Pierre Habib une intuition originale et profonde: le rôle décisif de la vaporisation du fluide interstitiel, qui explique les vitesses extrêmes atteintes par certains glissements, intuition dont Jean Sulem évoque la nombreuse descendance.

Gulihem Devèze illustre le début de carrière de Pierre Duffaut, jeune ingénieur au Service Géologie d'EDF, par deux exemples qui soulignent le rôle essentiel de la reconnaissance hydrogéologique pour les barrages : les lits fossiles du Drac et de la Loire, sur les aménagements de Montpezat et Monteynard. Il les éclaire par de nombreux documents inédits, dont certains de la main de Pierre Duffaut, dont l'approche morphologique demeure un outil efficace d'implantation des campagnes de reconnaissance. Il rappelle aussi le rôle de Pierre Duffaut globetrotteur, infatigable propagandiste de la Nouvelle Méthode Autrichienne pour le soutènement des tunnels et de l'utilisation des solutions souterraines, notamment pour les centrales nucléaires, que Jean Piraud évoque également.

Claude Louis évoque la passion de Pierre Duffaut pour La Houille Blanche et les barrages, le rôle de la filiation paternelle, les vocations d'ingénieur qu'il a suscité, ses relations fécondes avec Pierre Habib et Pierre Londe, sa vocation d'organisateur, le réseau international qu'il avait su créer - et Malpasset, sous un angle différent de celui de Jean Piraud. Il détaille aussi ses contributions à la conception de nombreux barrages dans le monde.

Ce sont deux thèmes - les centrales souterraines et Malpasset - que met aussi en exergue Jean Piraud. Il livre une 
analyse éclairante des circonstances de la catastrophe, qui n'omet ni les déchirements des concepteurs ni les dimensions organisationnelles, juridiques et administratives. Il rappelle le rôle de Pierre Habib et Pierre Duffaut dans l'organisation d'un colloque, sous l'égide du CFMR et du CFGI, à l'occasion du cinquantième anniversaire de la catastrophe. Il évoque les «trois Pierre», en associant Pierre Londe, et en montrant l'apport singulier de Pierre Duffaut, dont la vaste culture le plaçait à l'intersection des disciplines utiles à la compréhension de la catastrophe.

Luc Thorel rappelle la contribution décisive de Pierre Habib à l'éclaircissement de la notion d'effet d'échelle et à ses conséquences pour la réalisation des maquettes en géotechnique, dont celles de fondations et de tirants d'ancrage, et particulièrement des maquettes embarquées en centrifugeuse, outil dont il a été l'ardent et décisif promoteur du développement en France.

Ce sont deux hommes de grande culture scientifique et technique que la journée commune et ce numéro spécial saluent. Quelques traits ne ressortiront pas nécessairement de ces actes en forme de florilège. Il faut rappeler que Pierre Habib, directeur du LMS, y a fait conduire des expérimentations décisives au service de la défense de notre pays.
Pierre Habib et Pierre Duffaut ont été au cœur de l'émergence internationale de la mécanique des roches comme discipline scientifique autonome - Pierre Habib en a présidé la Société Internationale, et Pierre Duffaut a organisé les premiers échanges entre laboratoires allemands, britanniques, suisses et français. Il faut aussi dire leur amour de la langue française - Pierre Habib a été le défenseur inébranlable de l'usage du français dans les instances internationales; la RFG a recueilli son article inattendu et éclairant Toponymie et géotechnique; Pierre Duffaut, coordinateur d'une collection en langue française qui n'a pas d'équivalent, savait donner aux nouveaux concepts de nos disciplines des appellations à la fois justes et élégantes (Claude Louis rappelle qu'il est sans doute le créateur du mot tunnelier). Ceux qui en ont été les plus proches témoigneront volontiers de leur attention aux travaux de plus jeunes chercheurs et des encouragements qu'il savait leur prodiguer.

Pierre Bérest

Ancien directeur du LMS, École polytechnique. Palaiseau cedex, France pierre.berest@polytechnique.edu 